\title{
Relação entre coeficiente de recessão e índice de precipitação antecedente em bacias embutidas
}

Relation between recession coefficient and antecedent precipitation index in nested catchments

Submissāo 25/09/2018

Revisão: $10 / 12 / 2018$

Aceite: $23 / 12 / 2018$

\section{1 - Leonardo Batistel \\ 2 - Fernando Grison \\ 3 - Aline de Almeida Mota}

\section{RESUMO}

O trabalho investigou o comportamento do coeficiente de recessão $(K)$ do hidrograma e sua relaçáo com as condiçôes de umidade antecedente de três bacias hidrográficas embutidas. $\mathrm{O} K$ foi estimado por meio de 2 métodos: (i) ajuste exponencial dos segmentos de recessão do hidrograma para cada um dos eventos identificados; (ii) aplicação do método da tabulação para obtençáo das curvas de recessáo mestre $(M R C s)$ para cada bacia e ano. Não foi verificada diferença significativa entre os coeficientes $K$ médios obtidos pelos 2 métodos. Os valores característicos de $K$ se mostraram superiores a 0,999, implicando em baixa taxa de decaimento da recessão, ou seja, as bacias apresentam potencialidade de armazenamento de água. Calculou-se o índice de precipitação antecedente $(A P I)$ para os intervalos de 3, 7, 14, 21 e 30 dias e realizou-se análises estatísticas de regressão simples e multivariada. Os valores de API ficaram entre 0 e $30 \mathrm{~mm}$, sendo que sua variabilidade aumenta quanto menor a bacia. Náo foi encontrada uma relação estatística significativa entre $A P I$ e $K$, sugerindo que este não é o parâmetro mais adequado para explicar o comportamento da recessáo de acordo com condiçôes de umidade das bacias.

Palavras-chave: bacia hidrográfica do rio dos Bugres, curva de recessão mestre, retenção de água, umidade antecedente.

\section{ABSTRACT}

The work investigated the behavior of the recession coefficient $(K)$ of the hydrograph and its relation with the antecedent humidity conditions of three nested catchments. The $K$ was estimated by applying two methods: (i) exponential adjustment of the recession segments of the hydrograph for each of the identified events; (ii) application of the tabulation method to obtain the master recession curves $(M R C s)$ for each basin and year. No significant difference was observed between the average $K$ coefficients obtained by the 2 methods. The characteristic values of $K$ were higher than 0.999 , implying a low rate of decay of the recession, that is, the basins present water storage potentiality. The antecedent precipitation index (API) was calculated for the 3, 7, 14, 21 and 30-days intervals and statistical analyzes of simple and multivariate regression were performed. The $A P I$ values were between 0 and $30 \mathrm{~mm}$, and its variability increases the smaller the catchment. No significant statistical relationship was found between $A P I$ and $K$, suggesting that this is not the most adequate parameter to explain the behavior of the recession with the basin moisture conditions.

Keywords: Bugres river catchment, master recession curves, water retention, antecedent moisture.

\section{INTRODUÇÃO}

Segundo Hornberger et al. (1998), no século XX observou-se um grande aumento da utilização da água, bem como o aumento no risco de sua contaminaçáo.
Tal aumento da utilizaçáo da água implica na necessidade de realização de estudos em hidrologia aplicada, em busca de conhecimentos científicos para propor 
orientaçóes para o planejamento e gestão adequados dos recursos hídricos (CHOW; MAIDMENT; MAYS, 1988). Diante disso, é fundamental a instalação de bacias experimentais e sistemas de monitoramento hidrológico para o enriquecimento do banco de dados hidrológicos (MOTA et al., 2017).

Apesar da Hidrologia ser uma ciência amplamente desenvolvida, ainda um dos seus grandes desafios é compreender a origem e o comportamento das partes que compóem a água em um rio, ou canal natural. Ou seja, separar os escoamentos superficiais, subsuperficiais e de base, ou subterrâneos. Tallaksen (1995), em seu trabalho de revisão de métodos de análise de escoamento de base, verificou a existência de dificuldades em distinguir as diferentes origens de um fluxo. $\mathrm{O}$ escoamento de base representa a porção do fluxo proveniente da água subterrânea, podendo ser examinado a partir da análise de curvas de recessão de hidrogramas.

A análise de curvas de recessão é um método frequentemente utilizado na prática hidrológica, pois propicia a obtenção de vários resultados e interpretaçóes (GREGOR; MALÍK, 2012a). Por exemplo, sua análise fornece informaçóes sobre as características de retenção da bacia e de armazenagem subterrânea (WITTENBERG, 1994; TALLAKSEN, 1995).

$\mathrm{O}$ ajuste de uma curva de recessão propicia o cálculo do parâmetro de recessão, ou coeficiente de recessão $(K)$. Este parâmetro representa a condutividade hidráulica e a porosidade do solo da bacia de drenagem (SUJONO; SHIKASHO; HIRAMATSU, 2004).

Sujono, Shikasho e Hiramatsu (2004) ressaltam que a elevada variabilidade encontrada no comportamento dos segmentos individuais de recessão é um problema na determinação do coeficiente K. Para superar esse problema, os autores propóem o ajuste da curva de recessão mestre do escoamento de base. Essa curva é expressa por um gráfico característico de fluxos de recessão compilados pela sobreposição de um conjunto com diversas das curvas de recessão (HORTON, 1933).

A análise de hidrogramas e o ajuste de curvas de recessão para estimativa do coeficiente de recessão se mostram como importantes ferramentas para a compreensão do comportamento da água na bacia hidrográfica. Costa e Bacellar (2010) investigaram as condiçôes dos aquíferos de nove microbacias e de duas bacias maiores por meio de estimativas de coeficiente de recessão. As possíveis relações existentes entre o coeficiente de recessão e as características e condiçôes da bacia hidrográfica podem fornecer informaçóes que auxiliam no adequado planejamento e gestão de recursos hídricos.

O Índice de Precipitação Antecedente (Antecedent Precipitation Index - $A P I)$ é um parâmetro que representa a umidade armazenada dentro de uma bacia de hidrográfica antes da ocorrência de um evento de precipitaçáo. Pode ser definido como uma soma ponderada dos valores diários de precipitação e é utilizado como um índice de umidade do solo (LINSLEY; KOHLER, 1951). Segundo Boughton e Droop (2003), o API foi desenvolvido a partir da necessidade de prever inundaçóes. Por isso, seu uso mais frequente é na previsão de inundaçôes, mas também é um importante fator na determinação da geração de sedimentos nas bacias hidrográficas (FEDORA; BESCHTA, 1989). Sittner, Schauss e Monro (1969) utilizaram o API como base de um modelo de chuva-vazão para a simulação contínua de hidrograma sintético.

$\mathrm{O}$ API pode estar relacionado com vários parâmetros hidrológicos. Mota (2012), encontrou uma relação entre o API de 21 dias e o tempo de concentração de uma pequena bacia hidrográfica pequena bacia hidrográfica experimental, caracterizada por vegetação secundária de Floresta Ombrófila Mista. Sagara (2001), estimou o $A P I$ de vários eventos de chuva em uma pequena bacia hidrográfica experimental de 18,12 hectares, com predominância de atividade de reflorestamento. Os valores de API foram correlacionados com valores de coeficiente de recessão, obtendo a existência de uma relação $A P I$ e $K$ com $\mathrm{R}^{2}=0,788$.

No intuito de melhorar a compreensão da dinâmica dos compartimentos que compóe os escoamentos de água de uma bacia hidrográfica esse trabalho buscou investigar a relaçáo entre o escoamento de base e as condiçóes de armazenamento de água na bacia. Para isso, realizaram-se estimativas de coeficientes de recessão que foram relacionados com sazonalidade e condiçốes de umidade antecedente da bacia, utilizando $A P I$. 


\section{METODOLOGIA}

\section{Área de estudo}

A Bacia Hidrográfica do Rio dos Bugres (BHRB) possui área de $66,41 \mathrm{~km}^{2}$, está localizada no norte do estado de Santa Catarina e inserida em sua totalidade no município de Rio Negrinho - SC (Figura 1).

Em sua maior parte, a área da BHRB é caracterizada por Floresta Ombrófila Mista $(57,11 \%)$ e reflorestamento $(22,98 \%)$, aparecendo também uso de agricultura, pastagem e área urbanizada $(5,96 \%, 3,26 \%$ e 1,01\%, respectivamente) (GRISON; MOTA; KOBIYAMA, 2014).

Os solos predominantes da BHRB são Cambissolos que compreendem solos minerais, não hidromórficos, com horizonte B incipiente bastante heterogêneo, tanto no que se refere à cor, espessura e textura, quanto no que diz respeito à atividade química da fração argila e saturaçáo por bases (EMBRAPA, 2004).

A geologia tem características bastante variadas, porém existe a predominância de rochas sedimentares, tipo arenito e folheto, os quais são resultantes da deposição de extensas e espessas sequências de sedimentos predominantemente finos (SANTA CATARINA, 1986).

\section{Dados de vazáo}

Grison (2013) e Grison, Mota e Kobiyama (2014) e estudaram a BHRB e nela instalaram 11 pontos de monitoramento fluviométrico no rio dos Bugres, denominados de RB01 a RB11 no sentido do exutório para montante. Destes, apenas em RB01, RB10 e RB11 foi instalado monitoramento automático de nível d'água (Figura 1). Além disso, junto ao ponto
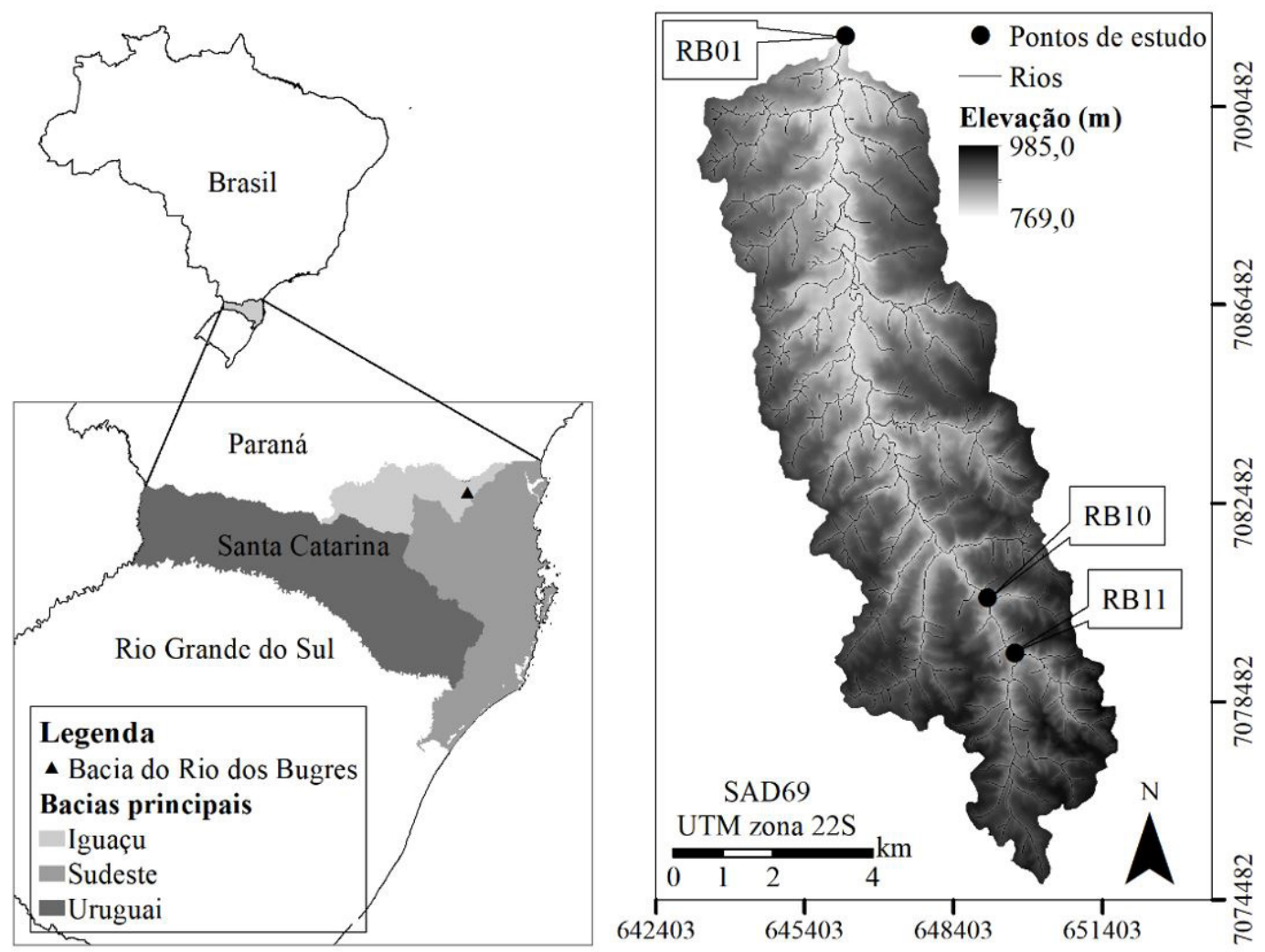

Figura 1 - Localização da bacia hidrográfica do Rio dos Bugres 
RB01 foi instalada uma estação automática para monitoramento pluviométrico. Os dados da BHRB foram monitorados entre os anos de 2011 a 2014, com intervalo de 10 minutos.

Para realização desse trabalho foram utilizadas as séries de dados pluviométricos e fluviométricos desses pontos com monitoramento automático. Portanto, foram analisadas as bacias com exutórios nos pontos RB01, RB10 e RB11, com área de drenagem de $66,41,11,45$ e $6,95 \mathrm{~km}^{2}$, respectivamente

Para as estimativas de vazóes $(Q)$ foram utilizadas as curvas-chave construídas por Grison (2013) e modificadas por Cardoso (2013), conforme as equaçóes de 1 a 8 e seus respectivos limites de aplicabilidade: i. RB01:

$$
\begin{aligned}
& C T \leq 1,16 m Q=3,27 \cdot C T^{2,60} \\
& 1,16<C T \leq 2,21 m Q=3,11 \cdot C T^{2,93} \\
& 2,21<C T \leq 2,25 m Q=1,34 \cdot C T^{3,98}
\end{aligned}
$$

ii. RB10:

$$
\begin{aligned}
& C T \leq 0,98 m Q=2,51 \cdot C T^{2,04} \\
& 0,98<C T \leq 1,22 m Q=3,94 \cdot C T^{-1,43} \\
& 1,22<C T \leq 1,35 m Q=2,03 \cdot C T^{2,59}
\end{aligned}
$$

iii. RB11:

$$
\begin{aligned}
& C T \leq 0,76 m Q=1,61 \cdot C T^{18,4} \\
& 0,76<C T \leq 1,22 m Q=1,79 \cdot C T^{2,00}
\end{aligned}
$$

onde $Q$ é a vazão, em $\mathrm{m}^{3} / \mathrm{s}$; e $C T$ é a cota linimétrica, em $\mathrm{m}$.

Ressalta-se que os dados de vazão para cotas maiores que os intervalos apresentados não foram considerados devido ao aumento da incerteza nas estimativas. Entretanto, isso não acarretou em prejuízos nas análises para o presente trabalho, pois para as análises das curvas de recessão dos hidrogramas não são necessários dados de vazão de pico.

A Figura 2 apresenta um fluxograma das principais atividades do trabalho, que estão complementarmente a esta etapa explicadas na sequência.

\section{Separaçáo de eventos}

A partir da construção de hidrogramas foi possível identificar os picos de vazáo e separar os segmentos que correspondem à recessão.

Para facilitar a separação das recessóes, utilizou-se o software HydroOffice que possibilita a análise dos dados hidrológicos e exportação dos dados separados para outros softwares de análise. Seguiram-se as recomendaçóes expressas por Gregor e Malík (2012b), no manual de utilização do software.

\section{Análise da recessão de hidrogramas}

Para uma melhor compreensão dos processos que influenciam no escoamento de base os segmentos das curvas de recessão foram analisados individualmente e coletivamente.

A Figura 3 apresenta a curva de recessão em um hidrograma resultante de um evento de precipitação. Também estão representados o segmento de recessão e o ponto de inflexão.

\section{Análise individual}

Para análise individual das curvas de recessão, primeiramente foi identificado o ponto de inflexão $(I)$ do hidrograma (Figura 3), ponto que separa o escoamento superficial do de base. Para isso, foi utilizado o método do cruzamento de retas (KOBIYAMA et al., 2006). A partir do segmento de recessão do hidrograma em escala logarítmica foram desenhadas duas retas descontínuas observando a tendência da curva (Figura 4). O ponto de interseção delas determina o fim do escoamento superficial.

A partir do ponto de inflexão, os dados de vazáo foram ajustados utilizando a Equação 9 a seguir:

$Q=Q 0 \cdot K^{t}$

onde $Q$ é a vazão no tempo, $Q_{0}$ é a vazão no início da recessão, $K$ é o coeficiente de recessão e $t$ é o tempo.

O coeficiente de recessão $K$ é expresso pela Equação 10:

$K=e^{-f c}$

onde $f c$ é a constante de decaimento da vazão. 


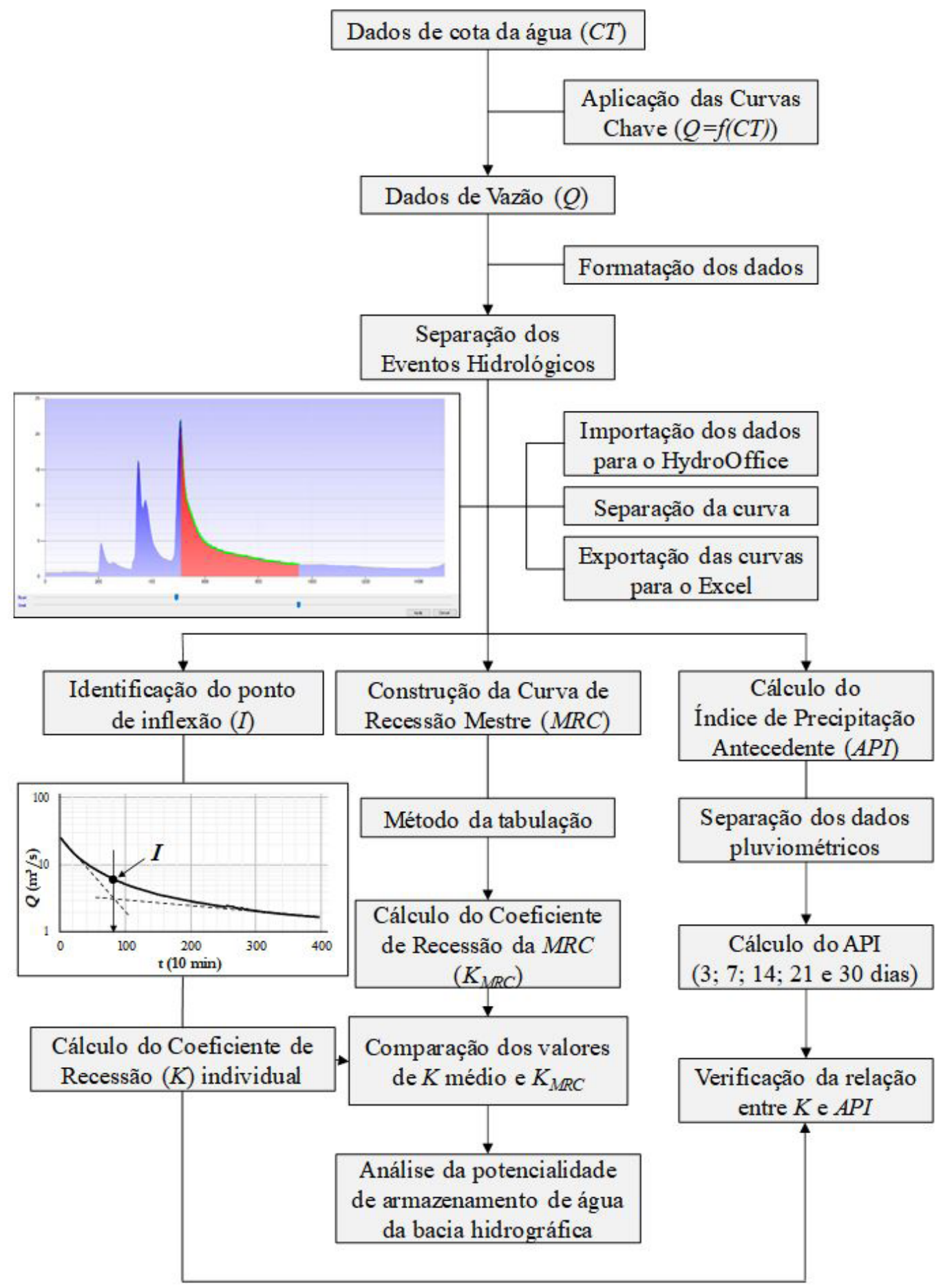

Figura 2 - Fluxograma de atividades 
A partir do ponto de inflexão, os dados foram ajustados exponencialmente, conforme a Figura 5.

Devido à incerteza na determinação do exato local do ponto de inflexão e para verificar a confiabilidade do intervalo de dados ajustados no processo de obtenção

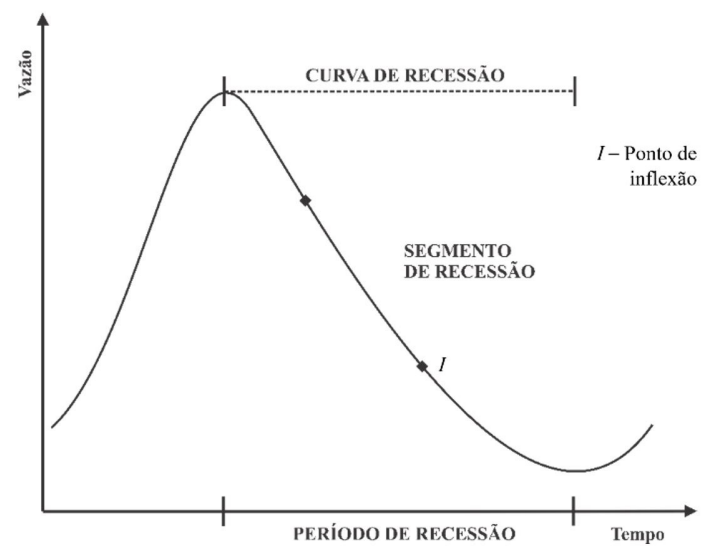

Figura 3 - Representação da curva de recessão, período e segmento (Adaptado de TALLAKSEN, 1995).

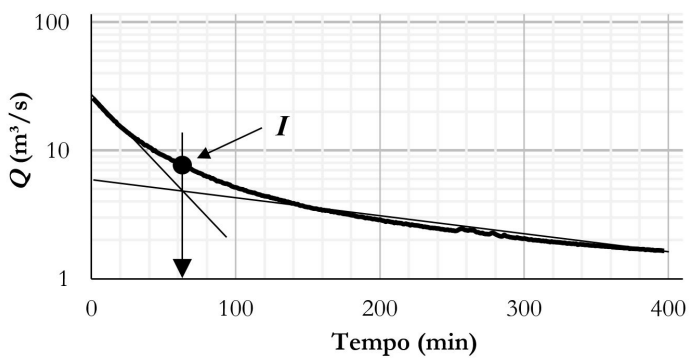

Figura 4 - Definição do ponto de inflexão $(I)$ na curva de recessão.

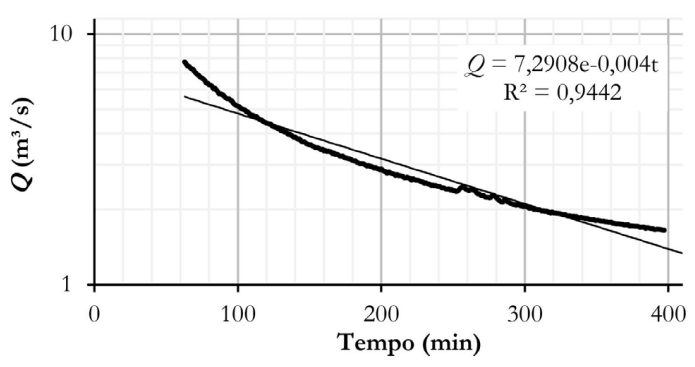

Figura 5 - Segmento de recessão ajustado exponencialmente do coeficiente de recessão $K$, realizaram-se variaçóes na quantidade de dados compreendidos no ajuste, de forma a averiguar possíveis mudanças nos valores de $K$. Constatou-se que pequenas variaçóes no intervalo de dados não influenciaram nos parâmetros obtidos do ajuste, ou seja, pequenos deslocamentos na definição do ponto de inflexáo (adotando o ponto $I$ a partir de um pequeno intervalo de tempo antes ou depois do local definido no método de cruzamento de retas) e o fim do segmento de recessão não interferiram nas estimativas de $K$. Para os pontos de estudo RB01, RB10 e RB11 foram utilizados 135, 97 e 84 eventos de vazão, respectivamente.

A fim de verificar alguma mudança de comportamento dos coeficientes de recessão, os eventos foram separados por estação do ano e calculados coeficiente médios para cada série de dados.

\section{Análise coletiva}

A análise coletiva das curvas de recessão consiste em unir vários segmentos de recessão do hidrograma de forma a construir uma única curva. Essa curva é conhecida como curva de recessão mestre (Master Recession Curve - MRC).

Sujono, Shikasho e Hiramatsu (2004) ressaltam a existência de diversos métodos que podem ser aplicados para construir a curva mestre. Segundo Hall (1968), três métodos bastante arbitrários são comumente utilizados para analisar o hidrograma e estimar a curva de recessão mestre: Matching Strip Method, Método da Correlação e Método da Tabulação.

Para a construção da curva de recessão mestre nesse trabalho utilizou-se o método da tabulação (TOEBES; STRANG, 1964). Neste método, encontra-se o maior valor inicial entre todos os segmentos de recessão e considera-o como valor inicial da curva recessão mestre.

Os outros segmentos são entâo combinados, sendo a $M R C$ igual à média dos valores dos segmentos, conforme exemplifica a Figura 6.

Em seguida, a curva de recessão mestre é representada num gráfico como uma única curva de recessão. O coeficiente de recessão $K$ é determinado da mesma forma que na análise individual da curva de recessão.

Nesse trabalho foram construídas uma MRCs a cada ano de dados, conforme a quantidade dos dados 


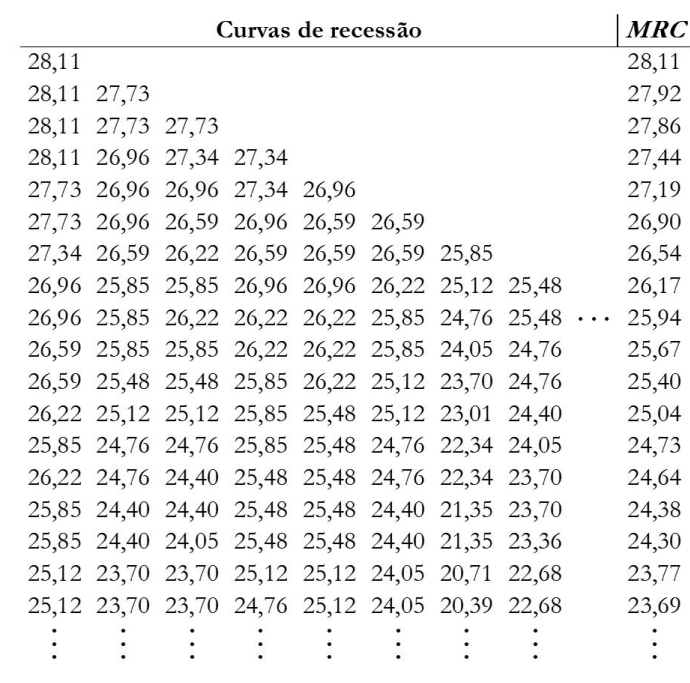

Figura 6 - Exemplo de construção da $M R C$ pelo método da tabulação.

obtidos em RB01, RB10 e RB11. Para a bacia RB01 foi possível obter as MRCs para todos os anos da série de dados. No entanto, por falhas nas séries de dados, em RB10 e RB11, só foi possível obter as MRCs para os anos 2012 e 2013. Após a obtenção do coeficiente $K$ das $M R C s$, efetivou-se a comparação dos valores encontrados e uma análise sobre a confiabilidade e adequação dos métodos aplicados.

\section{Índice de Precipitação Antecedente (API)}

Segundo Linsley e Kohler (1951), o API é definido por uma equação do tipo:

$$
A P I=\sum_{i=1}^{n}\left(b_{i} \cdot P_{i}\right)
$$

onde $A P I$ é o Índice de Precipitação Antecedente, $P_{i}$ é a precipitação acumulada em $i$ dias anteriormente ao evento considerado e $b_{i}$ é uma constante dada em função do tempo expressa por $b_{i}=1 / i$ e $n$ é o número de termos arbitrariamente selecionados.

Buscando-se estabelecer uma possível relação de influência das condiçóes de umidade antecedente da BHRB sobre o comportamento das suas recessóes de fluxo de água em RB01, RB10 e RB11, valores de $A P I$ foram estimados para os intervalos de
3, 7, 14, 21 e 30 dias. Para isso, foram utilizados os dados de chuva medidos na estação pluviométrica localizada junto ao ponto RB01.

Realizaram-se análises estatísticas de regressão linear simples utilizando o $A P I$ como a variável independente $(K=f(A P I))$ e análises de regressão linear multivariada, utilizando $A P I$ e $Q_{0}$ como variáveis independentes $\left(K=f\left(A P I, Q_{0}\right)\right)$ para todos os intervalos de APIs obtidos.

\section{RESULTADOS E DISCUSSÃO}

De maneira geral, as MRCs apresentam decaimento semelhantes, em todos os anos, sugerindo que os coeficientes de recessão também apresentem valores semelhantes (Figuras 7, 8 e 9). Observa-se também que os valores das vazóes em que as curvas se estabilizam tendem a aumentar de montante para jusante entre as bacias, ou seja, de RB11 para RB01. O que é esperado, tendo em vista que ocorre um aumento da área de drenagem entre os pontos que potencializa o armazenamento de água.

Vale destacar que as MCRs indicam que nas áreas de cabeceira da BHRB a retenção de água se mantém na maior parte do tempo abaixo de $0,5 \mathrm{~m}^{3} / \mathrm{s}$. Já na parte baixa da BHRB essa retenção se estabiliza entre 2 e $4 \mathrm{~m}^{3} / \mathrm{s}$, aproximadamente.

$\mathrm{Na}$ Tabela 1 são apresentados os valores médios anuais dos coeficientes de recessão das análises individuais $(\bar{K})$, os coeficientes de recessão das $M R C$ s $\left(K_{M R C}\right)$, juntamente com seus respectivos coeficientes de correlação médio anual $\left(\overline{R^{2}} \mathrm{e} R_{M R C}^{2}\right)$, para cada um dos pontos de estudo.

Observa-se que os valores obtidos para os coeficientes de recessão médios anuais se apresentam bastante similares em relação aos obtidos nas respectivas MRCs. Também, pode-se ressaltar que os valores dos coeficientes de correlação foram superiores a 0,9 , indicando que as curvas de recessão se ajustam bem ao modelo exponencial.

Os valores de $K$ se mostraram próximos a 1,0, indicando potencialidade de retenção de água na bacia hidrográfica, uma vez que aplicados na Equação 9 tem-se uma aproximação relevante entre os valores de $Q_{0}$ e de $Q$ em função do tempo.

Valores de $K$ próximos a 1,0 implicam em baixas taxas de decrescimento de fluxo, ou seja, as contribuiçóes do fluxo de base sofrem poucas alteraçóes ao longo do 


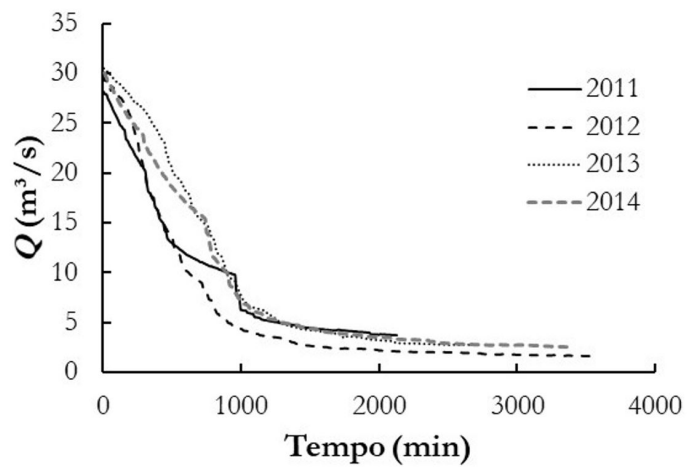

Figura 7 - Curvas de recessão mestre da bacia RB01.

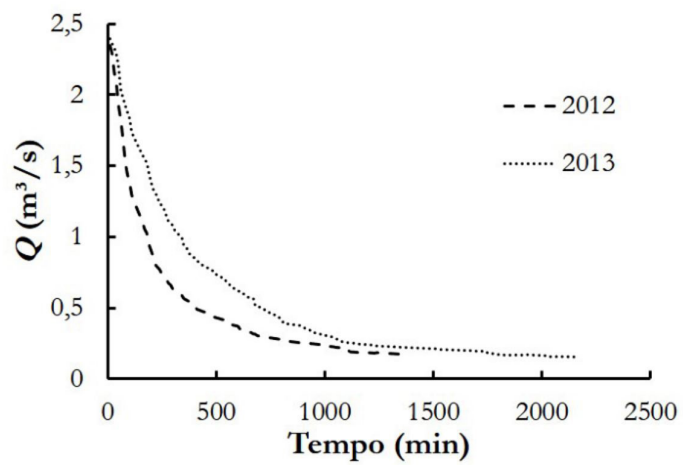

Figura 8 - Curvas de recessão mestre da bacia RB10.

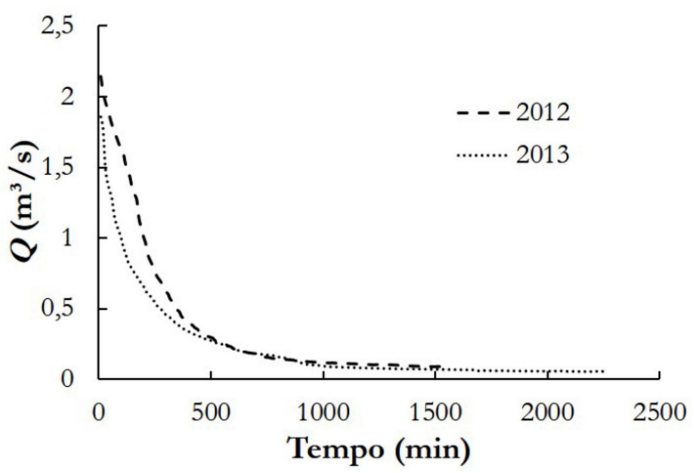

Figura 9 - Curvas de recessão mestre da bacia RB11.
Tabela 1 - Coeficientes de recessão médios e das $M R C s$

\begin{tabular}{ccccc}
\hline \multirow{2}{*}{ Ano $\downarrow$} & $\bar{K}$ & $\bar{R}^{2}$ & $K_{M R C}$ & $R_{M R C}^{2}$ \\
\cline { 2 - 5 } RB01 \\
\hline 2011 & 0,9997 & 0,93 & 0,9997 & 0,97 \\
2012 & 0,9997 & 0,92 & 0,9998 & 0,93 \\
2013 & 0,9998 & 0,95 & 0,9996 & 0,94 \\
2014 & 0,9998 & 0,95 & 0,9997 & 0,94 \\
\hline \multicolumn{5}{c}{ RB10 } \\
\hline 2011 & 0,9990 & 0,98 & - & - \\
2012 & 0,9992 & 0,97 & 0,9989 & 0,97 \\
2013 & 0,9995 & 0,94 & 0,9994 & 0,93 \\
2014 & 0,9995 & 0,97 & - & - \\
\hline \multicolumn{5}{c}{ RB11 } \\
\hline 2011 & 0,9994 & 0,98 & - & - \\
2012 & 0,9992 & 0,95 & 0,9991 & 0,91 \\
2013 & 0,9993 & 0,94 & 0,9995 & 0,90 \\
2014 & 0,9988 & 0,98 & - & - \\
\hline
\end{tabular}

tempo, tendendo a manter-se constantes. Dessa forma, pode-se afirmar que a reta de ajuste do escoamento de base tende a horizontalidade.

Costa e Bacellar (2010) estimaram valores do coeficiente $K$ para várias bacias brasileiras caracterizadas geologicamente por rochas ígneas e metamórficas (granitos, gnaisses e xistos). Os valores de $K$ para 9 bacias com área menor que $1 \mathrm{~km}^{2}$, utilizando dados diários, variaram entre 0,9598 e 0,9966. Já para 2 bacias com área de 116 e $312 \mathrm{~km}^{2}$ aproximadamente os valores de $K$ foram de 0,9958 e 0,9924 , respectivamente. Os coeficientes $K$ da BHRB são maiores, e uma possível explicação é a influência do tipo de rocha, já que as bacias possuem geologia tipo sedimentar que por ter maior porosidade, favorece $\mathrm{o}$ armazenamento de água e posterior escoamento para o canal fluvial.

\section{Análise de sazonalidade}

$\mathrm{Na}$ Figura 10 pode-se destacar que RB01 tem as menores variaçóes entre os quartis de todos os box-plots. A maior variação entre os coeficientes ocorre para RB10 no outono. Analisando-se juntamente todos os valores de $K$ em cada ponto de estudo, observa-se que de RB11 para RB01 (de montante para jusante na BHRB) a variação dos coeficientes 


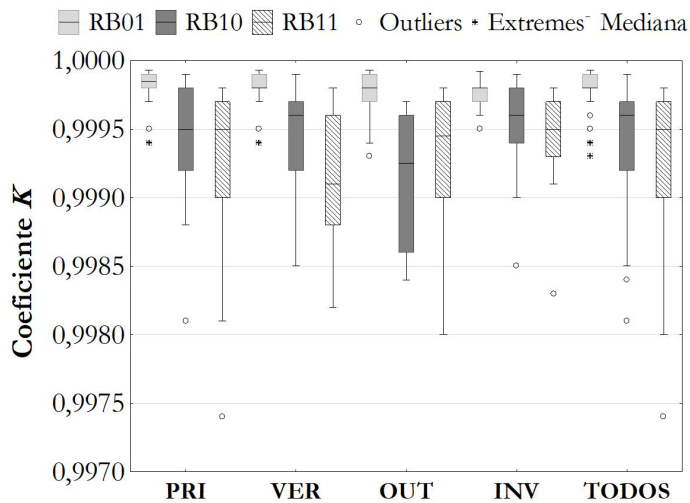

Figura 10 - Variação dos coeficientes de recessão das análises individuais por estaçóes do ano e nos 3 pontos de estudo (“TODOS").

diminui, ou seja, há uma tendência de que quanto maior a retenção e armazenamento de água de água na bacia o escoamento de base fique mais estável. A essa constatação pode ser adicionada a informação de que quanto mais estável for o escoamento de base, maior a sua permanência, conforme mostram as MRCs dos pontos de estudo (Figuras 7, 8 e 9).

\section{Relaçáo entre umidade antecedente e coeficiente de recessáo}

Os valores de $A P I$ se concentraram entre 0 e $30 \mathrm{~mm}$ nos intervalos analisados. Em geral, a variabilidade e os valores de $A P I s$ aumentam quanto menor a bacia, sendo que a diferença entre os valores para cada bacia é muito similar para cada intervalo considerado (Figura 11).

A Tabela 2 apresenta os coeficientes de correlaçáo obtidos para as regressóes simples (utilizando o $A P I$ como a variável independente, $K=f(A P I))$ e multivariada (utilizando $A P I$ e $Q_{0}$ como variáveis independentes, $\left.K=f\left(A P I, Q_{0}\right)\right)$.

As regressões apresentaram valores de coeficientes de correlação $\left(R^{2}\right)$ abaixo de 0,1 , em praticamente todas as análises efetivadas, implicando assim que não existe relação significativa entre os valores de $A P I$ sobre os valores de $K$ bem como entre os valores de $A P I$ e $Q_{0}$ sobre $K$.

Sagara (2001) correlacionou o coeficiente $K$ com API obtendo resultados bastante satisfatórios, com
RB01 RB10 RB11 - Outliers * Extremes Mediana

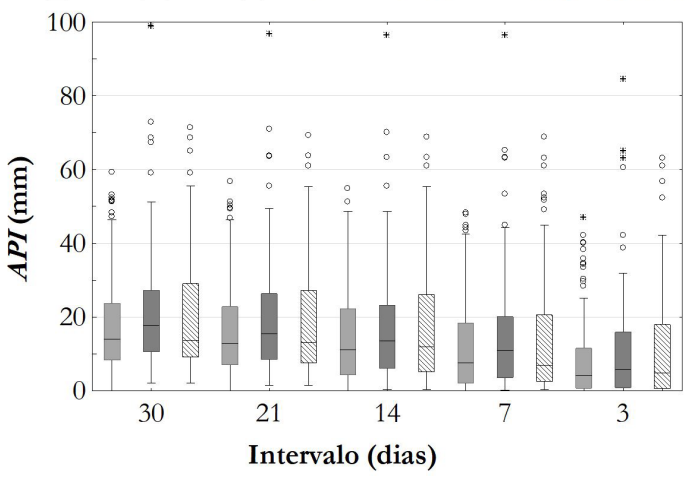

Figura 11 - Variação de APIs por pontos de estudo da BHRB.

Tabela 2 - Resultados das análises de regressão simples e multivariada

\begin{tabular}{cccc}
\hline \multirow{2}{*}{$A P I($ dias $) \downarrow$} & RB01 & RB10 & RB11 \\
\cline { 2 - 4 } & \multicolumn{2}{c}{$\mathrm{R}^{2}-$ Regressão Simples } \\
\hline $\mathbf{3}$ & 0,001522 & 0,003706 & 0,028234 \\
$\mathbf{7}$ & 0,000933 & 0,009107 & 0,051154 \\
$\mathbf{1 4}$ & 0,000073 & 0,023753 & 0,067628 \\
$\mathbf{2 1}$ & 0,000001 & 0,029649 & 0,081770 \\
$\mathbf{3 0}$ & 0,000008 & 0,029221 & 0,080673 \\
\hline \multicolumn{4}{c}{$\mathrm{R}^{2}-$ Regressão Multivariada } \\
\hline $\mathbf{3}$ & 0,078773 & 0,025085 & 0,030295 \\
$\mathbf{7}$ & 0,087970 & 0,038971 & 0,051458 \\
$\mathbf{1 4}$ & 0,100385 & 0,063053 & 0,067801 \\
$\mathbf{2 1}$ & 0,070254 & 0,072313 & 0,081822 \\
$\mathbf{3 0}$ & 0,068013 & 0,077034 & 0,080729 \\
\hline
\end{tabular}

coeficientes de correlação elevados, mostrando assim a existência de uma relação entre as condiçôes de umidade da bacia hidrográfica e o comportamento da recessão do fluxo dos eventos hidrológicos. Porém, pode-se atribuir tal diferenciação nos resultados de Sagara (2001) às características morfométricas e morfológicas da bacia hidrográfica, as quais se distinguem da BHRB. Pode-se ainda ressaltar sobre o intervalo de amostragem dos dados de vazão considerados nos estudos. Além disso, Sagara (2001) trabalhou com dados de vazóes diários e neste estudo utilizou-se de dados de vazóes com intervalos de 10 (dez) minutos. 


\section{CONCLUSÓES}

O conhecimento do coeficiente de recessão $K$ se mostra como uma importante ferramenta no processo de compreensão do comportamento da dinâmica da água na bacia hidrográfica, já que expressa características relacionadas aos estoques naturais de água que alimentam o fluxo dentro da bacia.

Os valores encontrados nas estimativas do coeficiente $K$ expressam grande potencial de armazenamento de água na BHRB, promovendo sua capacidade de ser uma bacia alternativa de captação de água para abastecimento público e outras atividades passíveis de outorga. Porém, pelas análises das $M C R s$, é preciso observar que nas áreas de cabeceira da BHRB o armazenamento de água se estabiliza com vazóes bem inferiores aquelas estimadas no exutório. Essa informação é importante ser considerada em projetos que visam a preservaçáo da quantidade de água da BHRB.

A construção da curva de recessão mestre por meio do método da tabulaçáo se mostrou adequada, uma vez que as $M C R s$ apresentaram coeficientes de recessão semelhantes aos valores médios obtidos pelas análises das curvas de recessão individuais.

A inexistência de uma relação entre $A P I$ e o coeficiente de recessão $K$ sugere que o comportamento do fluxo de base não é determinado pelas condiçōes de umidade antecedente da bacia hidrográfica, demonstrando assim que a vazão depende de outros fatores além da precipitação. Novas investigaçôes conexas ao comportamento do coeficiente de recessão se mostram necessárias, buscando estabelecer relaçôes do comportamento do fluxo de base na BHRB.

Deve-se salientar que a investigaçáo de outros parâmetros morfométricos e morfológicos da bacia hidrográfica buscando-se estabelecer relaçôes expressivas com o coeficiente de recessão é de suma importância.

\section{REFERÊNCIAS}

BOUGHTON, W.; DROOP, O. Continuous simulation for design flood estimation: a review. Environmental Modelling and Software, Amsterdam, v. 18, n. 4, p. 309-318, 2003.

CARDOSO, A. T. Estudo hidrossedimentológico em três bacias embutidas no município de Rio Negrinho - SC. 2013. 101 f. Dissertação (Mestrado em Engenharia Ambiental) - Programa de Pós-graduação em Engenharia Ambiental, Universidade Federal de Santa Catarina, Florianópolis, 2013.

CHOW, V. T.; MAIDMENT, D. R.; MAYS, L. W. Applied hydrology. New York: McGraw-Hill, 1988. 572 p. (McGraw-Hill Series in Water Resources and Environmental Engineering).

COSTA, F. M.; BACELLAR, L. A. P. Caracterização hidrogeológica de aquíferos a partir do fluxo de base. Revista Brasileira de Recursos Hídricos, Porto Alegre, v. 15, n. 3, p. 173-183, 2010.

EMBRAPA. Solos do Estado de Santa Catarina. Rio de Janeiro: EMBRAPA Solos, 2004. (Boletim de Pesquisa e Desenvolvimento, 46).

FEDORA, M. A.; BESCHTA, R. L. Storm runoff simulation using an antecedent precipitation index (API) model. Journal of Hydrology, Amsterdam, v. 112, n. 1-2, p. 121-133, 1989.

GREGOR, M.; MALÍK, P. Construction of master recession curve using genetic algorithms. Journal of Hydrology and Hydromechanics, Bratislava, v. 60, n. 1, p. 3-15, 2012a.

GREGOR, M.; MALÍK, P. RC 4.0 user's manual: software for water science. 2012b. 36 p. Disponível em: <http://hydrooffice.org/>. Acesso em: 21 jul. 2015.

GRISON, F. Estudo da geometria hidráulica do Rio dos Bugres, no município de Rio Negrinho - SC. 2013. 242 f. Tese (Doutorado em Engenharia Ambiental) - Programa de Pós-graduação em Engenharia Ambiental, Universidade Federal de Santa Catarina, Florianópolis, 2013.

GRISON, F.; MOTA, A. A.; KOBIYAMA, M. Geometria hidráulica de seções transversais do rio dos Bugres. Revista Brasileira de Recursos Hídricos, Porto Alegre, v. 19, n. 4, p. 205-213, 2014.

HALL, F. R. Base-flow recessions: a review. Water Resources Research, Washington, v. 4, n. 5, p. 973-983, 1968.

HORNBERGER, G. M. et al. Elements of physical hydrology. In: Groundwater hydrology. Baltimore: The Johns Hopkins University Press, 1998. 302 p.

HORTON, R. E. The role of infiltration in the hydrologic cycle. Transactions, Washington, v. 14, n. 1, p. 446-460, 1933.

KOBIYAMA, M. et al. Estimativa morfométrica e hidrológica do tempo de concentração na bacia do campus da UFSC, Florianópolis - SC. In: SIMPÓSIO DE RECURSOS HÍDRICOS DO SUL-SUDESTE, 1., 2006, Curitiba. Anais... Curitiba: UFPR, 2006. 11 p.

LINSLEY, R. K.; KOHLER, M. A. Predicting the runoff from storm rainfall. U.S. Weather Bureau, 1951. 10 p. (Research Paper, 34). 
MOTA, A. A. Tempo de concentração em pequena bacia experimental. 2012. 131 f. Dissertação (Mestrado em Engenharia Ambiental) Universidade Federal de Santa Catarina, Florianópolis, 2012.

MOTA, A. A. et al. Instalação de uma pequena bacia experimental florestal: estudo de caso da bacia do Rio Araponga. Engenharia Sanitaria e Ambiental, Rio de Janeiro, v. 22, n. 1, p. 73-80, 2017.

SAGARA, F. T. Estudo hidrológico de uma pequena bacia hidrográfica experimental no município de General Carneiro - PR, através de monitoramento e modelagem. 2001. 65 f. Dissertação (Mestrado) - Curso de Agronomia, Ciências Agrárias, Universidade Federal do Paraná, Curitiba, 2001.

SANTACATARINA. Gabinete de Planejamento e Coordenação Geral. Subchefia de Estatística, Geografia e Informática. Atlas de Santa Catarina. Rio de Janeiro: Aerofoto Cruzeiro, 1986. 173 p.
SITTNER, W. T.; SCHAUSS, C. E.; MONRO, J. C. Continuous hydrograph synthesis with an api-type hydrologic model. Water Resources Research, Washington, v. 5, n. 5, p. 1007-1022, 1969.

SUJONO, J.; SHIKASHO, S.; HIRAMATSU, K. A comparison of techniques for hydrograph recession analysis. Hydrological Processes, Chichester, v. 18, n. 3, p. 403-413, 2004.

TALLAKSEN, L. M. A review of baseflow recession analysis. Journal of Hydrology, Amsterdam, v. 165, n. 1-4, p. 349-370, 1995.

TOEBES, C.; STRANG, D. D. On recession curves, 1. Recession equations. Journal of Hydrology, New Zeland, v. 3, n. 2, p. 2-15, 1964.

WITTENBERG, H. Nonlinear analysis of flow recession curves. In: FRIEND: Flow Regimes from International Experimental and Network Data. 1994. p. 61-67. (IAHS Publication, 221).

Contribuição do autor:

Organizou o banco de dados, fez a separação dos eventos de vazão, estimou os coeficientes de recessão e os índices de precipitação antecedente. Escreveu o trabalho.

Fernando Grison

Contribuição do autor:

Instalou o sistema de monitoramento hidrológico e realizou a coleta dos dados em campo. Idealizou o objetivo do trabalho.

Orientou e revisou a escrita do trabalho.

Aline de Almeida Mota

Universidade Federal da Fronteira Sul. aline.mota@uffs.edu.br

Contribuição do autor:

Instalou o sistema de monitoramento hidrológico e realizou a coleta dos dados em campo. Trabalhou nas análises das correlaçóes do coeficiente de recessão com $A P I$ e na elaboração de alguns gráficos. Revisou a escrita do trabalho. 